\title{
Retrofittable T-ray antireflection coatings
}

\author{
W. Withayachumnankul, B. M. Fischer, S. P. Mickan, and D. Abbott \\ Centre for Biomedical Engineering and School of Electrical \& Electronic Engineering, \\ The University of Adelaide Adelaide, SA 5005, Australia
}

\begin{abstract}
Terahertz time-domain spectroscopy (THz-TDS) is able to extract optical or dielectric properties of materials, whether in the solid, liquid, or gas phase, in the T-ray frequency region. Spectroscopy of a liquid or gas often requires a receptacle to confine the sample. In order to allow T-rays to probe the sample effectively, the receptacle must have T-ray transparent windows. However, even though windows are transparent to T-rays, attenuation exists, because of multiple reflections at air-window and window-air interfaces, which accounts for a major energy loss. Due to the recent emergence of T-ray technology, there has been very little work carried out to-date on the reduction of reflection losses. This paper analyses the reduction of T-ray reflection loss by means of an antireflection coating. Because T-ray wavelengths are much larger than visible wavelengths, the antireflection layer thickness for T-rays is much larger than the usual optical case. This creates an interesting opportunity for retrofittable antireflection layers in T-ray systems. In the experiment, a coating material made from polyethylene sheets is applied onto the surfaces of a silicon window. The coated window shows enhancement of the transmittance within a range of frequencies.
\end{abstract}

Keywords: Antireflection coating, terahertz time-domain spectroscopy, T-rays

\section{INTRODUCTION}

Typically, when the electromagnetic field propagates through a slab of material, it experiences two modes of amplitude attenuation - one is the absorption loss and the other is the reflection loss. The absorption loss is due to bulk material, inside which molecules absorb electromagnetic energy converting it to vibrational motion or heat. This type of loss exponentially depends on the extinction coefficient, $\kappa$, of material and the propagation length, $l$. It is possible to control, i.e. decrease or increase, the absorption loss to some extent through temperature manipulation, since the extinction coefficient is thermally-dependent.

Another cause of amplitude attenuation is reflection loss. In this case, the wave energy is not converted into other energy forms, but rather diverted to another direction. When the field encounters an interface between two media, part of the field is reflected off a surface. The reflection direction and the reflection amplitude are predicted by Snell's law and Fresnel's law, respectively. The important parameters in both laws is the refractive index of the material. In case of the normal incidence at an air-sample interface, the reflection coefficient is given by $\rho=(n-1) /(n+1)$ and thus the reflectance is $\mathcal{R}=(n-1)^{2} /(n+1)^{2}$, where $n$ is the index of refraction of the sample. As $n$ increases from unity to infinity, the reflectance increases from zero to unity. The reflectance of some common materials, used for T-ray windows is illustrated in Figure 1. The figure shows the reflectance accounting for only one surface. When two surfaces, i.e. the entry and exit faces of a slab, are considered the reflectance increases to $2 \mathcal{R}-\mathcal{R}^{2}$.

Obviously, amplitude attenuation due to the reflection loss becomes dominant for transparent materials. This leads to the requirement for reducing such loss, in particular when a transparent material functions as window. Through antireflection coating on a window surface, the propagation direction can be controlled in such a way that it reduces the reflectance and enhances the transmittance. The underlying mechanism of an antireflection coating is the interference formed by reflections inside the coating layer(s). A simple illustration showing possible antireflection coating options is given in Figure 2.

W. Withayachumnankul, Email: withawat@eleceng.adelaide.edu.au;

D. Abbott, Email: dabbott@eleceng.adelaide.edu.au

Micro- and Nanotechnology: Materials, Processes, Packaging, and Systems III, edited by Jung-Chih Chiao, Andrew S. Dzurak, Chennupati Jagadish, David Victor Thiel, Proc. of SPIE Vol. 6415, 64150N, (2006) - 0277-786X/06/\$15 - doi: 10.1117/12.695949

Proc. of SPIE Vol. $641564150 \mathrm{~N}-1$ 


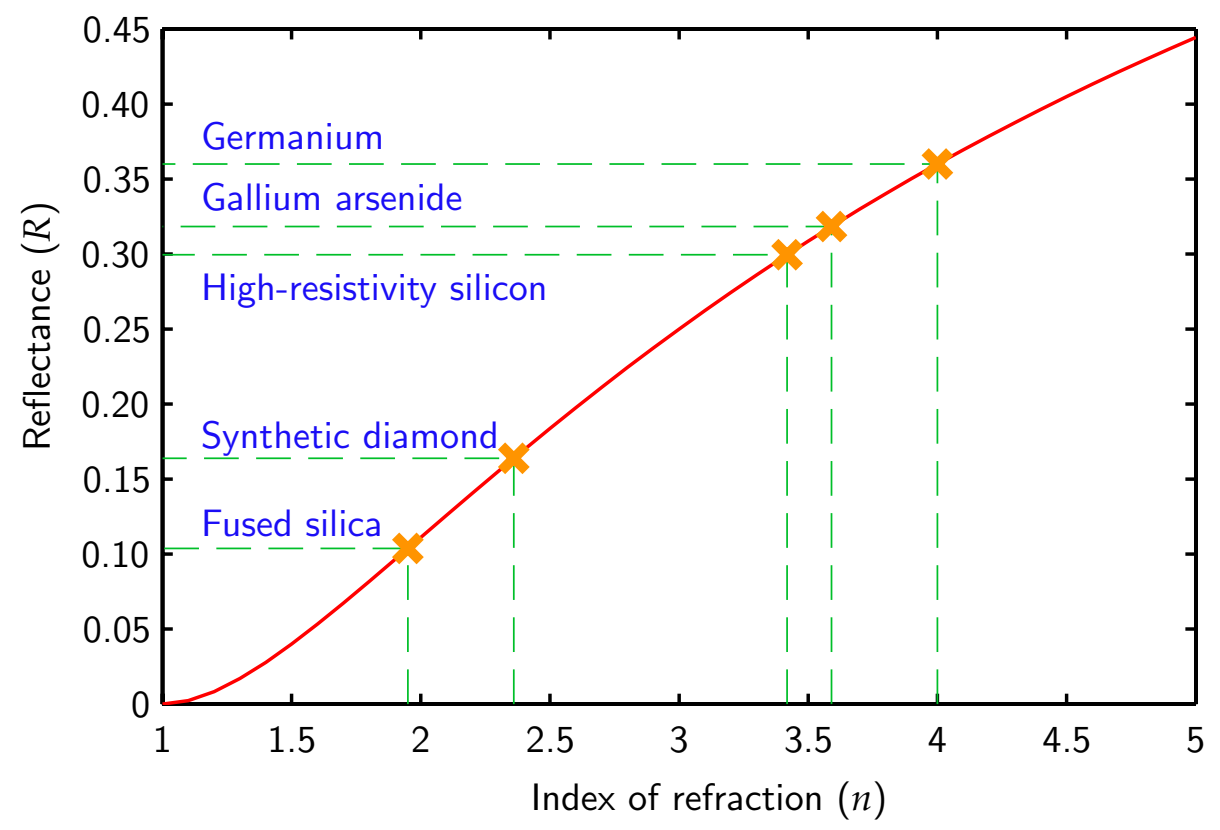

Figure 1. Reflectance of common materials, for T-rays at normal incidence. The reflectance is calculated from $\mathcal{R}=$ $(n-1)^{2} /(n+1)^{2}$ for some common materials used as window or substrate. The indices of refraction of these materials are for T-ray radiation.

This work presents the retrofittable antireflection coating, by which the window transmittance is increased for selected T-ray frequencies. An example structure composed of a T-ray transparent window, made from a highresistivity silicon wafer, and a coating, made from common polyethylene films is demonstrated. The structure is characterised by THz-TDS, which has a reliable frequency range between 0.2 and $1.0 \mathrm{THz}$. To the best knowledge of the authors, this work is the first to intensively study an antireflection coating in this frequency range. This range is of particular interest for biosensing. ${ }^{1}$

The paper is organised as follows. Section 2 briefly reviews some of the work on antireflection coatings, which operate at high T-ray frequencies, overlapping with the far-infrared band. The theory of quarter-wave antireflection coatings is given in Section 3, which leads to theoretical models of the reflectance and transmittance in Section 4. Section 5 covers some common T-ray transparent materials, which can either be used as windows or coatings depending on their optical and mechanical properties. Possible coating techniques for a T-ray window are summarised in Section 6, followed by a proposed retrofittable antireflection coating in Section 7. Prior to construction of coated windows, all the involving bulk materials are characterised by THz-TDS to find their optical properties, presented in Section 8. This follows by the results and discussion in Section 9 and 10, respectively.

\section{ANTIREFLECTION COATINGS FOR T-RAY RADIATION}

So far, characteristics and fabrication methods of T-ray antireflection windows have been reported, for example, as follows:

Armstrong and Low ${ }^{2}$ attached polyethylene films $(n=1.5)$ onto many types of substrates, including synthetic sapphire, silicon, crystalline quartz, and calcium fluoride $\left(\mathrm{CaF}_{2}\right)$, by means of thermal bonds. The film thicknesses are in the order of ten microns. The spectroscopic data in the $1.5 \mathrm{THz}$ to $15 \mathrm{THz}$ range showed an increase in the transmittance for every substrate.

Kawase and Hiromoto ${ }^{3}$ glued fused quartz $\left(n=2.0, \quad \alpha=1 \mathrm{~cm}^{-1}\right)$ onto one side of an intrinsic germanium (Ge: $n=4.0$ ) or gallium arsenide (GaAs: $n=3.6$ ) substrate, and then polished the quartz to a thickness of 


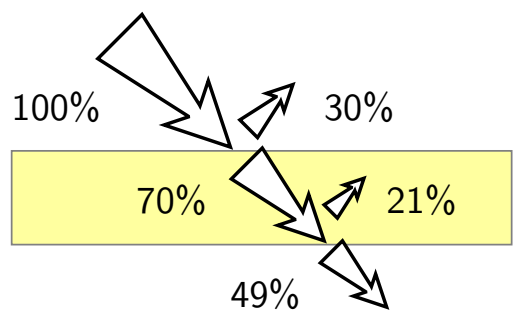

(a) Uncoated silicon window

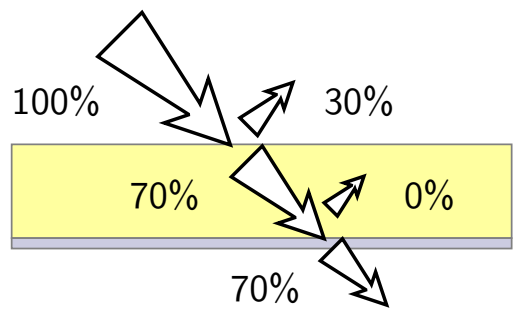

(c) Backside coated silicon window

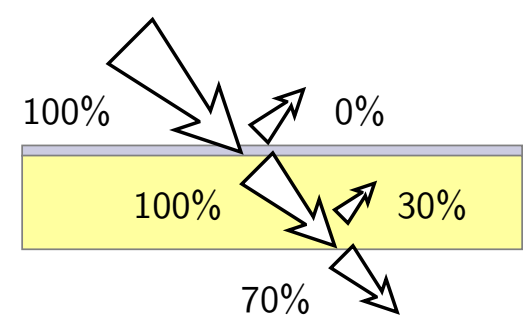

(b) Frontside coated silicon window

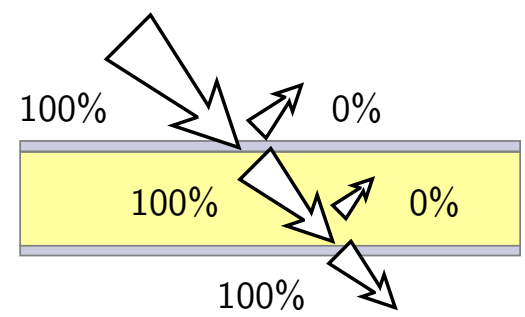

(d) Two-sided coated silicon window

Figure 2. Antireflection coatings for a silicon window. The given transmittance and reflectance percentages are for (i) a particular transmitting frequency, (ii) normal incidence, (iii) intrinsic silicon (no material absorption), (iv) an appropriate thickness and index of refraction of coating layer, (v) no internal reflections. The incident direction is tilted for visualization, and the arrows are not to scale.

approximately 20 microns. Measured by FTIR spectroscopy, the coated Ge and GaAs substrates exhibited 57percent transmittance at $1.74 \mathrm{THz}$, and 65 -percent transmittance at $1.43 \mathrm{THz}$, respectively. Later, Kawase et al. $^{4}$ glued fused quartz onto two sides of the Ge substrate to confirm reproducibility. This configuration raised the transmittance to 90 percent.

Englert et al. ${ }^{5}$ applied low-density polyethylene sheets (LDPE: $n=1.52, \alpha=66.8 \times 10^{-2} \mathrm{~cm}^{-1}, l=18.5 \mu \mathrm{m}$ ) to both sides of a wedged silicon window in vacuum atmosphere, and heated the LDPE-coated window up to promote durability. This structure attained upto 90-percent transmittance at $2.5 \mathrm{THz}$. The coated window was used in aircraft allowing highly-efficient narrowband remote sensing of far-infrared radiation.

Gatesman et al. ${ }^{6}$ deposited parylene-C $\left(n=1.62, \alpha=11 \mathrm{~cm}^{-1}, l=24.0 \mu \mathrm{m}\right)$ or parylene-D $(n=1.62, \alpha=$ $11 \mathrm{~cm}^{-1}, l=26.5 \mu \mathrm{m}$ ) onto both sides of each silicon substrate. The parylene-C and parylene-D coated substrates gave the transmittance of 90 percent at $1.9 \mathrm{THz}$ and $1.7 \mathrm{THz}$, respectively. The authors noted that parylene coating thickness is not feasible below $1 \mathrm{THz}$.

Hosako $^{7}$ deposited a layer of silicon dioxide $\left(\mathrm{SiO}_{2}, n=2.10, l=20.9 \mu \mathrm{m}\right)$ on Ge substrate by the plasmaenhanced chemical vapour deposition (CVD) method. The transmittance of the substrate at $1.8 \mathrm{THz}$ was increased to $55 \%$. Hosako ${ }^{8,9}$ extended the previous work to a two-period coating structure, each period of which contains amorphous $\mathrm{Si}$ and $\mathrm{SiO}_{2}$. The coated germanium had the transmittance of over $90 \%$ along a broad frequency band, centered at $1.8 \mathrm{THz}$.

Recently, a few papers reported studies on antireflection coatings with ultrafast T-ray systems. For example, Löffler et al. ${ }^{10}$ employed THz-TDS to characterize a silicon substrate coated with a mixture of paraffin wax and silicon powder on a single side. The authors only demonstrated an amplitude spectrum of the sample, and a time-resolved signal was omitted. Furthermore, it was claimed that the index of refraction of the mixture was controllable by varying the mixture ratio, but there was no report on this index nor the coating thickness.

Another paper reported by Biber et al. ${ }^{11}$ involved an artificial antireflection coating, operating with ultrafast T-ray systems. A silicon window was etched on both surfaces, forming grids of an artificial dielectric with depth of quarter wavelength. Inspected by THz-TDS, this structure provides relatively broadband high transmittance 
over 430-670 GHz. However, the principle underlying this technique is based on interference induced by a 2dimensional periodic structure, and is beyond the scope of this work that focuses on homogeneous antireflection coatings.

It would appear that almost all previous research addressed the designs and analyses of antireflection windows in the mid T-ray frequency region, i.e. from $1.5 \mathrm{THz}$ to tens of terahertz. However, these designs are inappropriate for modern ultrafast T-ray biosensing systems, which operate in the frequency range of 0.1 to $2.0 \mathrm{THz}$ or higher. In addition, most prior measurements were carried out via conventional FTIR spectroscopy, which delivers only transmittance magnitude spectra.

\section{QUARTER-WAVE ANTIREFLECTION COATING}

Figure 3 shows the case of a single coating layer on a window where $l$ is the coating thickness, and $n_{0}, n_{a}$, and $n_{s}$ are the indices of refraction of air, coating material, and substrate, respectively. The window is assumed to be semi-infinite so that there is no reflection from the back surface. Through the characteristic matrix, the optimum coating parameters, $l$ and $n_{a}$, required for zero reflectance at a particular frequency can be derived.

When the field direction is normal to the surface, from the characteristic matrix method, ${ }^{12}$ the transmission and reflection functions of coating layer, independent of the polarization, are

$$
H_{t}(\omega)=\frac{2 n_{a} n_{0}}{n_{a}\left(n_{0}+n_{s}\right) \cos \left(\omega n_{a} l / c\right)+i\left(n_{0} n_{s}+n_{a}^{2}\right) \sin \left(\omega n_{a} l / c\right)},
$$

and

$$
H_{r}(\omega)=\frac{n_{a}\left(n_{0}-n_{s}\right) \cos \left(\omega n_{a} l / c\right)+i\left(n_{0} n_{s}-n_{a}^{2}\right) \sin \left(\omega n_{a} l / c\right)}{n_{a}\left(n_{0}+n_{s}\right) \cos \left(\omega n_{a} l / c\right)+i\left(n_{0} n_{s}+n_{a}^{2}\right) \sin \left(\omega n_{a} l / c\right)},
$$

respectively.

Hence, the reflectance of the coating layer equals

$$
\mathcal{R}=\left|H_{r}(\omega)\right|^{2}=\frac{n_{a}^{2}\left(n_{0}-n_{s}\right)^{2} \cos ^{2}\left(\omega n_{a} l / c\right)+\left(n_{0} n_{s}-n_{a}^{2}\right)^{2} \sin ^{2}\left(\omega n_{a} l / c\right)}{n_{a}^{2}\left(n_{0}+n_{s}\right)^{2} \cos ^{2}\left(\omega n_{a} l / c\right)+\left(n_{0} n_{s}+n_{a}^{2}\right)^{2} \sin ^{2}\left(\omega n_{a} l / c\right)},
$$

and according to the law of energy conservation (assuming no absorption in the coating material), the transmittance is

$$
\mathcal{T}=1-\mathcal{R}=\frac{4 n_{0} n_{s} n_{a}^{2}}{n_{a}^{2}\left(n_{0}+n_{s}\right)^{2} \cos ^{2}\left(\omega n_{a} l / c\right)+\left(n_{0} n_{s}+n_{a}^{2}\right)^{2} \sin ^{2}\left(\omega n_{a} l / c\right)} .
$$

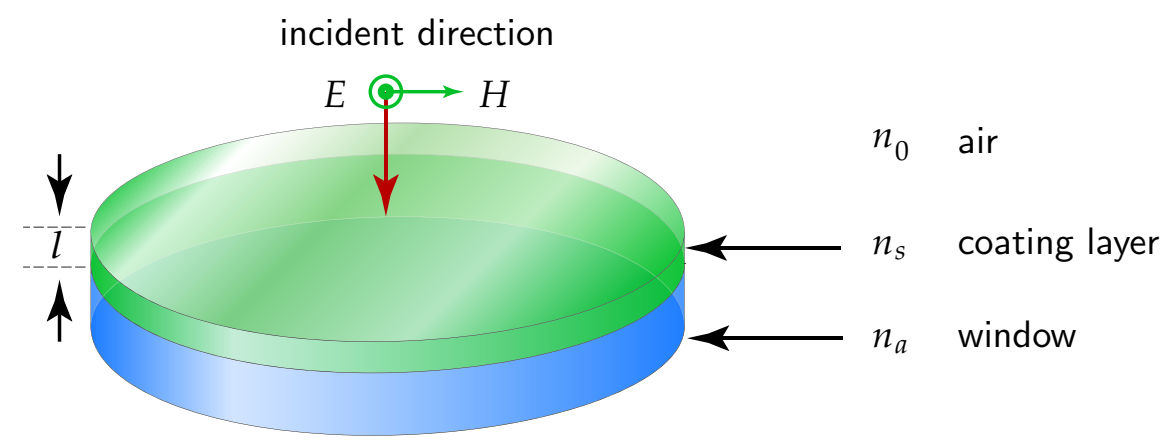

Figure 3. Antireflection coated window. The single coating layer has the index of refraction of $n_{a}$ and the thickness of l. The reflectance in Equation 3 is considered for a case that the field direction is normal to a surface of semi-infinite window. 
At $\omega n_{a} l / c=m \pi, m \in\{0,1,2, \ldots\}$, the transmittance becomes minimum and the reflectance becomes maximum, or

$$
\begin{aligned}
\mathcal{T}_{\min } & =\frac{4 n_{0} n_{s}}{\left(n_{0}+n_{s}\right)^{2}}, \\
\mathcal{R}_{\max } & =\frac{\left(n_{0}-n_{s}\right)^{2}}{\left(n_{0}+n_{s}\right)^{2}} .
\end{aligned}
$$

These expressions equal the transmittance and reflectance at an air-substrate interface without a coating material (Fresnel's law).

On the other hand, the reverse extrema occur at $\omega n_{a} l / c=(2 m+1) \pi / 2, m \in\{0,1,2, \ldots\}$, or

$$
\begin{aligned}
\mathcal{T}_{\max } & =\frac{4 n_{0} n_{s} n_{a}^{2}}{\left(n_{0} n_{s}+n_{a}^{2}\right)^{2}}, \\
\mathcal{R}_{\min } & =\frac{\left(n_{0} n_{s}-n_{a}^{2}\right)^{2}}{\left(n_{0} n_{s}+n_{a}^{2}\right)^{2}} .
\end{aligned}
$$

Thus, at the angular frequency $\omega$ the transmittance is unity and the reflectance is zero when

$$
n_{a}^{2}=n_{0} n_{s}
$$

This condition implies that, provided that the field is incident in free space, where $n_{0}=1$, when a coating material has the index of refraction equal to a square root of the index of refraction of the substrate, or

$$
n_{a}=\sqrt{n_{s}},
$$

the reflectance is zero at the frequency

$$
f_{c}=\frac{c}{4 n_{a} l_{a}}
$$

and its odd harmonics. Usually, the optimal frequency $f_{c}$ is pre-determined, and the coating thickness $l_{a}$ is selected according to Equation 9.

In a real situation Equation 8 cannot be satisfied due to a limited number of appropriate coating materials. Thus, a material with the closest index value must be adopted. With such an unmatched index value, the optimal frequency is still under control given that the coating thickness satisfies Equation 9.

It should be noted that Equation 9 can be rewritten in terms of optical thickness as

$$
n_{a} l_{a}=\frac{c}{4 f_{c}}=\frac{\lambda_{c}}{4} .
$$

The optical thickness of coating layer is a quarter of the wavelength. Thus this kind of coating is the so-called quarter-wave antireflection coating.

The effect of a quarter-wave antireflection coating can be visualised as follows. When the field travelling in a low index layer is reflected off by a higher index layer, its phase is shifted by $180^{\circ}$, or a half wavelength. In case of antireflection window, $n_{0}<n_{a}<n_{s}$, and therefore, the field reflected at either air-coating or coating-window interface will have a half wavelength phase shift. Furthermore, the field traversing the quarter-wave coating layer back and forth will have an additional phase shift of a half wavelength, resulting in a full wavelength phase shift, when it re-emerges into the air. The field reflected from the air-coating interface with a half wavelength phase shift will then destructively interfere with the field reflected from the coating-window interface with a full wavelength phase shift. 


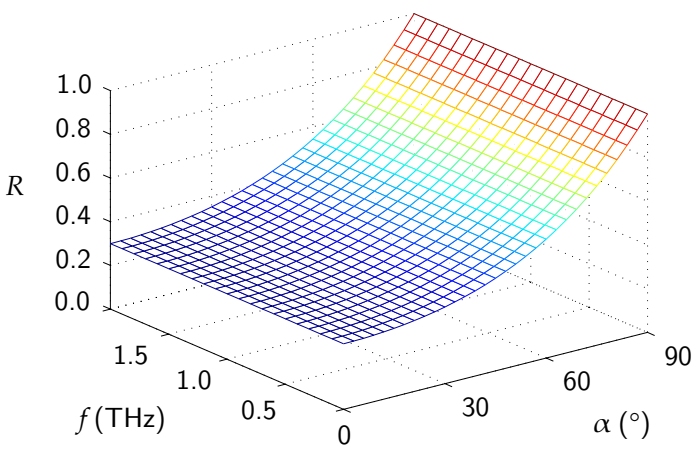

(a) Uncoated silicon, TE wave

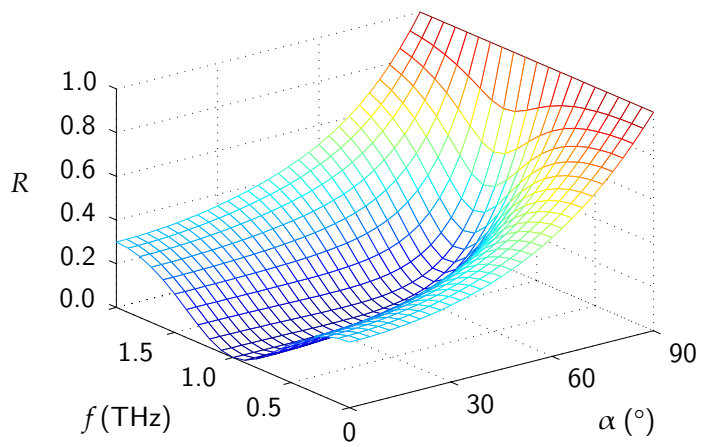

(c) Coated silicon, TE wave

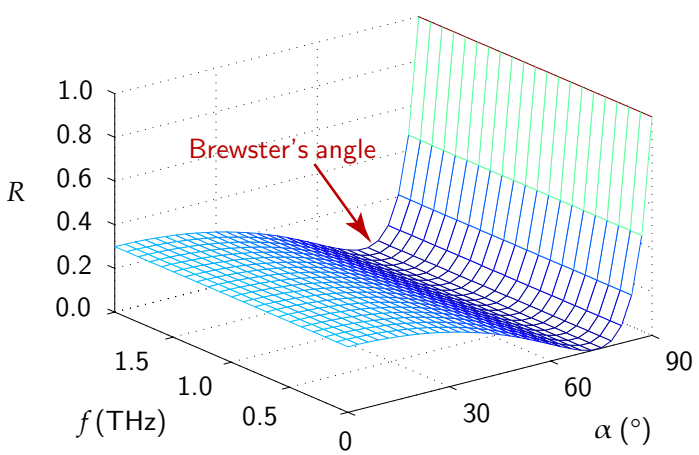

(b) Uncoated silicon, TM wave

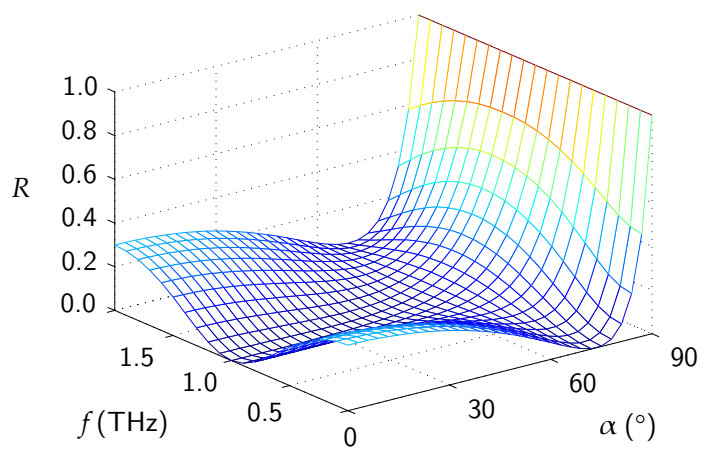

(d) Coated silicon, TM wave

Figure 4. Reflectance at the air-silicon interface. The reflectance is meshed versus the T-ray frequency, $f$, and the incident angle, $\alpha$. The coating material is set to have an index of refraction of $n_{a}=\sqrt{n_{\mathrm{Si}}}=1.85$, and the thickness is quarter of $1-\mathrm{THz}$ wavelength or $l_{a}=c /\left(4 n_{a} \times 10^{12}\right)=40.56$ microns. Brewster's angle for a silicon-air interface at $\alpha_{\text {Brewster }}=73.69^{\circ}$ can be seen in both cases of a $T M$ wave.

\section{REFLECTANCE AND TRANSMITTANCE MODELS}

\subsection{Reflectance of quarter-wave coated surface}

The coating layer influences the field propagation from lower to higher index material and vice versa in a similar way. This can be demonstrated by the reflectance models in Figures 4 and 5, for which the propagation direction is from air to a silicon window and from a silicon window to air, respectively. These reflectances are calculated using the characteristic matrix method. Only the effect at a air-silicon or silicon-air interface is taken into account without considering reflections inside a silicon slab. A coating material has the exact index value of $\sqrt{n_{\mathrm{si}}}$, and the thickness of a quarter of $1-\mathrm{THz}$ wavelength. The plots vary the frequency and the incident angle, and also the polarization ( $T E$ or $T M$ wave).

Consider Figure 4 for which the field propagation direction is from air to a silicon window. In the case of an uncoated window in Figure 4 (a) and (b), the reflectance is constant over the frequency range for a particular incident angle. For a TM wave there is a Brewster's angle, at which the reflectance is zero. When the window is coated with a perfectly conditioned material, the reflectances, as shown in Figure 4 (c) and (d), become zero at a selected frequency (1.0 THz in this case), and have low regions, for both $T E$ and $T M$ waves. However, the general reflectance characteristic of a coated window is still similar to that of an uncoated one. One feature that is hardly observable from the model reflectance for the coated window is that, as the incident angle increases from the normal, the optimal frequency (where the reflectance becomes zero) is lower. This is because longer apparent optical axis in a coating layer can accommodate longer wavelength.

A similar effect occurs when the field direction is from a coated window to air, as shown in Figure 5 (c) and (d). The reflectance is zero at a desired frequency and lowered at both sides. However, in this case the zero 


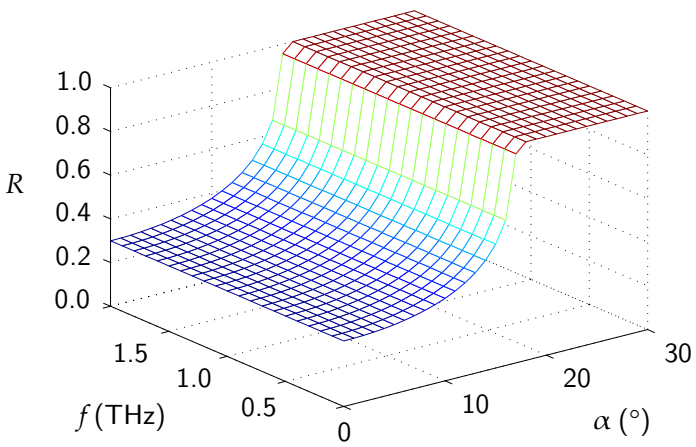

(a) Uncoated silicon, TE wave

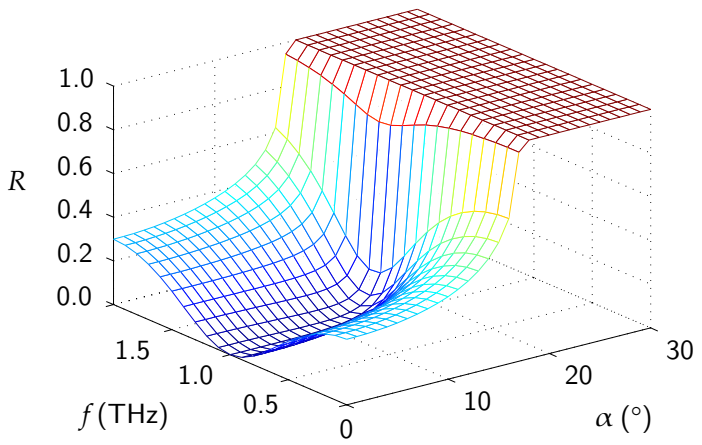

(c) Coated silicon, TE wave

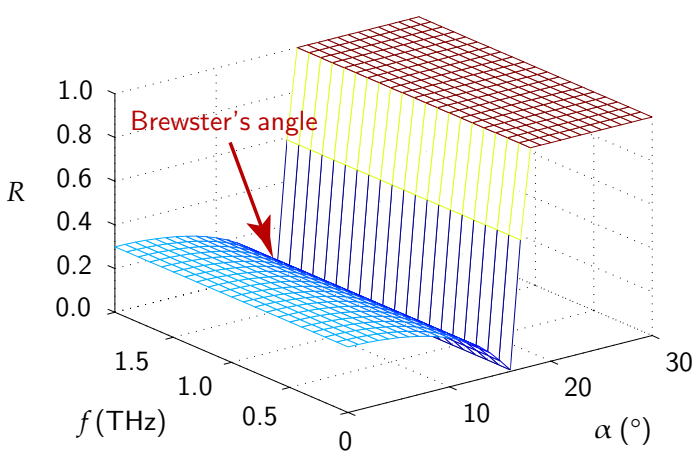

(b) Uncoated silicon, TM wave

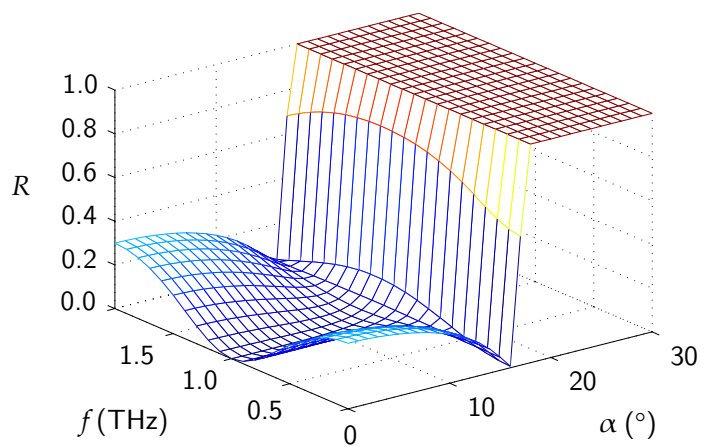

(d) Coated silicon, TM wave

Figure 5. Model reflectance at silicon-air interface. All parameters are identical to those used in Figure 4, excepting that the field direction here is from silicon to air. A critical angle at $\alpha_{\text {Critical }}=17.01^{\circ}$ and total internal reflection beyond the critical angle can be seen in all cases.

reflectance is limited by total internal reflection at and beyond the critical angle. The total internal reflection blocks the field transmission at all frequencies regardless of the polarization or the existence of coating layer.

Nevertheless, these model reflectances are considered at a single side of a window. In fact there are two effects involved in the real situation - the effect of Fabry-Pérot reflections, induced by a window, which cause fringes in the spectrum, and the effect of reflectance at the exit side of the window. These effects will be incorporated into a calculation, based on the characteristic matrix method, and will be shown in the next subsection.

\subsection{Transmittance of coated system}

Transmittance of a coated system, which includes results from the front and back coatings and the parallel surfaces of a window, is shown in Figure 6. The window and the coating layers in this example have the same parameters as those used in Figure 4. However, in this subsection the simulation focuses on the transmittance at normal incidence.

For the case when a silicon window is uncoated, the window transmittance is shown in Figure 6 (a). The fringes in the transmittance (green solid line) are caused by internal reflections between two parallel surfaces of the window. If these reflections are removed, the transmittance will be flattened over the frequency range (red dot line). When the window is coated on one side, the transmittance at a desired frequency (1.0 THz in this case) is enhanced from 0.5 to 0.7, as shown in Figure 6 (b). Obviously, the fringes at $1.0 \mathrm{THz}$ are reduced, as a result of the coating layer. When a window has coating layers on both surfaces, the transmittance at $1 \mathrm{THz}$ is further enhanced from 0.7 to unity, as depicted in Figure 6 (c).

In the real situation, the fringes that plague a spectrum are hard to remove if data is measured with conventional frequency-domain spectroscopy. However, with THz-TDS this seems to be possible, since THz-TDS 


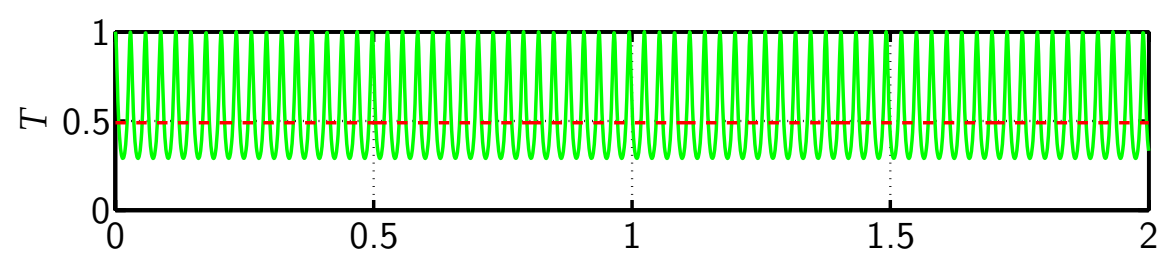

(a) Uncoated silicon window

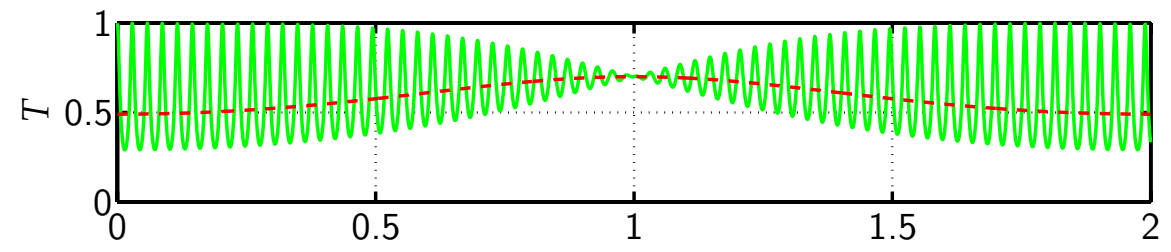

(b) One-side coated silicon window

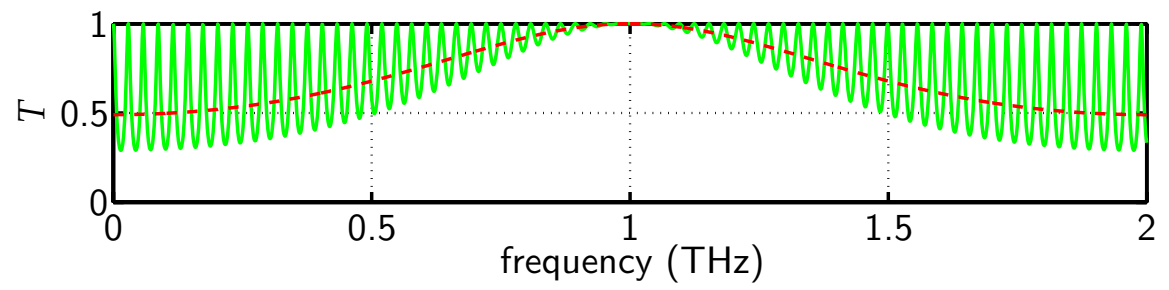

(c) Two-side coated silicon window

Figure 6. Transmittance of silicon window. The transmittance is plotted against the T-ray frequency. The thickness of silicon window is set to $1.5 \mathrm{~mm}$. The coating material is set to have the index of refraction $n_{a}=\sqrt{n_{\mathrm{Si}}}=1.85$, and the thickness is a quarter of $1-\mathrm{THz}$ wavelength or $l_{a}=c /\left(4 n_{a} \times 10^{12}\right)=40.56$ microns. (Green, solid) the transmittance incorporating reflections within silicon window, (Red, dotted) the transmittance with no reflections within a silicon window.

transmits a series of short pulses that is temporally recorded. Once a pulse encounters internal reflections and takes a longer path, it will be delayed, and can be easily removed in the time domain if pulse duration is short, relative to the reflection path.

\section{TRANSPARENT MATERIALS FOR ANTIREFLECTION WINDOWS}

It has been shown that, in the operation of antireflection windows, the interference mechanism dominates the frequency response, given that each material is non-dispersive and non-absorptive in the T-ray frequency band. A limited number of T-ray near-transparent materials have been reported thus far. Those include silicon and some low-loss polymers. Fortunately, the low absorption property of these materials implies the frequency dependence of index of refraction is small, i.e. non-absorption implies non-dispersion. ${ }^{13}$ Some of the common transparent materials usually found in T-ray components are discussed below. Optical constants of these and other low-loss materials for T-ray frequencies were intensively reviewed in Lamb ${ }^{14}$ and Simonis ${ }^{15}$ and references therein.

\subsection{Silicon}

Silicon plays an important role as the bulk material for T-ray components, such as lenses, beam splitters, or windows. In addition to its desirable physical and chemical properties, silicon's exceptional transparency and non-dispersive properties, at T-ray frequencies, are the most attractive in general. Its optical properties are shown in Table 1 in comparison to other common materials. A high-resistivity $(>10 \mathrm{k} \Omega \mathrm{cm})$ and high-purity silicon crystal, grown by the float-zone (FZ) method, shows, below $2 \mathrm{THz}$, an absorption coefficient lower than ${ }^{16,17}$ $0.05 \mathrm{~cm}^{-1}$ and a constant index of refraction of ${ }^{17} 3.418 \pm 0.001$. Moreover, the optical isotropy of silicon allows flexible orientation, regardless of the polarization of the incident T-rays and the crystal orientation. ${ }^{17}$ 
Table 1. The data shown are indices of refraction, $n$, and absorption coefficients, $\alpha$, measured at room temperature. The data are compiled from (a) Loewenstein et al. ${ }^{18}$ (below $2.1 \mathrm{THz}$ ), (b) Grischkowsky et al. ${ }^{17}$ (below $2.0 \mathrm{THz}$ ), and (c) Fischer $^{19}$ (below $2.8 \mathrm{THz}$ ). The numerical discrepancies are possibly due to differences in manufacturing and testing processes. Empty entries mean that data are unavailable.

\begin{tabular}{ccccccc}
\hline \multirow{2}{*}{ Material } & \multicolumn{2}{c}{ (a) } & \multicolumn{3}{c}{ (b) } & \multicolumn{1}{c}{ (c) } \\
\cline { 2 - 7 } & $n$ & $\alpha\left(\mathrm{cm}^{-1}\right)$ & $n$ & $\alpha\left(\mathrm{cm}^{-1}\right)$ & $n$ & $\alpha\left(\mathrm{cm}^{-1}\right)$ \\
\hline $\mathrm{SiO}_{2}$ (Fused silica) & & & 1.95 & $<8.0$ & 1.95 & $<15.0$ \\
High-resistivity silicon & 3.4175 & $<0.7$ & 3.418 & $<0.1$ & 3.41 & $<0.5$ \\
Synthetic diamond & & & & & 2.36 & $<0.5$ \\
Germanium & \multirow{2}{*}{4.006} & $<0.4$ & 4.0 & $<3.0$ & & \\
Gallium Arsenide & & & 3.59 & $<2.5$ & & \\
\hline
\end{tabular}

\subsection{Low-loss polymers}

Many types of low-loss polymers are available, and some are shown in Table 2. Although the indices of refraction of these polymers are very close to one another, each polymer type has its own advantages and disadvantages. Generally, low-loss polymers are superior to ultra-thin silicon with regard to conforming to different shaped surfaces. It is possible to mould the polymers to any shape; in particular, polymer films with thicknesses in the order of T-ray wavelengths are viable. However, unless the field direction is normal to a surface, almost all polymers exhibit birefringence, i.e. index of refraction that is dependent upon the incident direction and the polarization. ${ }^{20}$ Moreover, unlike semiconductors, the optical properties of polymers vary from sample to sample ${ }^{19}$ and are dependent on many manufacturing parameters. ${ }^{20}$ This variability suggests the need to measure the optical properties of each sample, ourselves, prior to its implementation.

In addition to those conventional polymers, two other types of polymers, including picarin $(n \approx 1.53)$ and cycloolefins $(n \approx 1.52)$, have been introduced recently. Unlike other polymers, these two materials have remarkable transparency in both T-ray and visible (including UV and near IR) frequency regions. This property is beneficial for an optical-pump/THz-probe experiment. However, their dielectric properties in the T-ray regime are still largely unreported in the literature. At falliing cost, these new polymers are commercially available in many forms.

Table 2. The data shown are indices of refraction, $n$, and absorption coefficients, $\alpha$, measured at room temperature. The

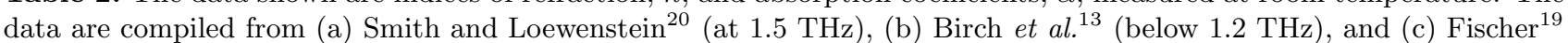
(below $2 \mathrm{THz}$ ). The discrepancies are possibly due to differences in manufacturing and testing processes. Empty entries mean that data are unavailable. LDPE - Low-density polyethylene. HDPE - High-density polyethylene. PTFE Polytetrafluoroethylene (Teflon@). PCTFE - Polychlorotrifluoroethylene. PS - Polystyrene.

\begin{tabular}{ccccccc}
\hline \multirow{2}{*}{ Material } & \multicolumn{2}{c}{$(\mathrm{a})$} & \multicolumn{2}{c}{$(\mathrm{b})$} & \multicolumn{3}{c}{$(\mathrm{c})$} \\
\cline { 2 - 7 } & $n$ & $\alpha\left(\mathrm{cm}^{-1}\right)$ & $n$ & $\alpha\left(\mathrm{cm}^{-1}\right)$ & $n$ & $\alpha\left(\mathrm{cm}^{-1}\right)$ \\
\hline LDPE & & & 1.5138 & $<0.5$ & & \\
HDPE & 1.518 & 1.4 & 1.5246 & $<0.5$ & & $<5.0$ \\
TPX® & 1.447 & 6.0 & 1.4600 & $<0.5$ & 1.43 & $<1.0$ \\
PTFE & & & 1.4330 & $<0.5$ & & $<1.5$ \\
PCTFE & & & & & $1.43,1.50$ & $<4.0$ \\
PS & & & 1.5912 & $<2.0$ & & \\
Cycloolefines & & & & $1.5 \sim 1.6$ & $<0.5$ \\
\hline
\end{tabular}




\section{COATING TECHNIQUES}

Coating techniques are commonly available to optical components, and coated optical components can be found in a number of daily-used devices, e.g. camera lenses, glasses, screen panels, and so on. Also the coating can occur in nature, and its effect, called thin-film interference, appears visible in colourful patterns. The samples of natural coating are soap bubbles, oil in water, and heated metals. For optical wavelengths, coating thicknesses of a fraction of micron satisfy the interference condition, and thin film coating technologies, such as a vacuum evaporation or a sputtering, and materials, such as $\mathrm{MgF}_{2}, \mathrm{SiO}$, or $\mathrm{ZnS}$, can support such degrees of thicknesses.

For longer T-ray wavelengths, much thicker coating layer(s), up to tens of microns, are necessary to satisfy the interference condition. The coating materials and techniques used in optics are not applicable to these wavelengths, ${ }^{21}$ because the materials themselves are not transparent when exposed to T-rays, and a deposited material cannot form a thick layer. Thus, researchers have studied new coating options suitable for T-rays.

Several methods for producing a thick coating layer have been reported, and some were mentioned briefly in Section 2. They can be categorized into two major types: material adhesion and material deposition.

Material adhesion involves preparing a thin film of material with suitable index of refraction, usually a polymer sheet, to a desired thickness. This film is applied to a substrate surface in vacuum to avoid bubbles between layers. They could be attached together by (i) a supporting frame with no adhesive, ${ }^{22,23}$ (ii) adhesive, ${ }^{3}$ or (iii) thermal bonds. ${ }^{2,5}$

Material deposition involves chemical reaction of suitable gases in a controlled chamber, which results in desired materials deposited onto a prepared substrate. The possible deposition techniques for T-ray filter fabrication are plasma-enhanced chemical vapour deposition $(\mathrm{CVD})^{7}$ and tetraethylorthosilicate-CVD. ${ }^{24}$

In general, selecting between material adhesion and material deposition has tradeoffs concerning fabrication complexity and layer coating quality. The deposition technique definitely requires complex equipment, and the deposition time is directly proportional to the coating thickness. However, the final result is durable and uniform even on a complex substrate surface. Material adhesion, in opposition, is much simpler in terms of fabrication, but a coating cannot be applied to an uneven surface. Furthermore, a coating is prone to peeling off a substrate unless heating is applied to the structure during fabrication. ${ }^{2,5}$ General comparisons between material adhesion and deposition are given in Table 3. In addition, comparisons among many specific methods can be found in Hosako. ${ }^{8}$

Table 3. Comparison of the micrometer-film fabrication methods. The comparisons are given in general terms, and the characters could be different from these for a particular method.

\begin{tabular}{lcc}
\hline Character & Adhesion & Deposition \\
\hline Proper coating materials & Limited & Less limited \\
Fabrication time & Short & Long \\
Fabrication temperature & Low & High \\
Fabrication equipment & Simple & Complicated \\
Residue stress & No & Yes \\
Durability & Low & High \\
Formability & No & Yes \\
Uniformity & Low & High \\
\hline
\end{tabular}




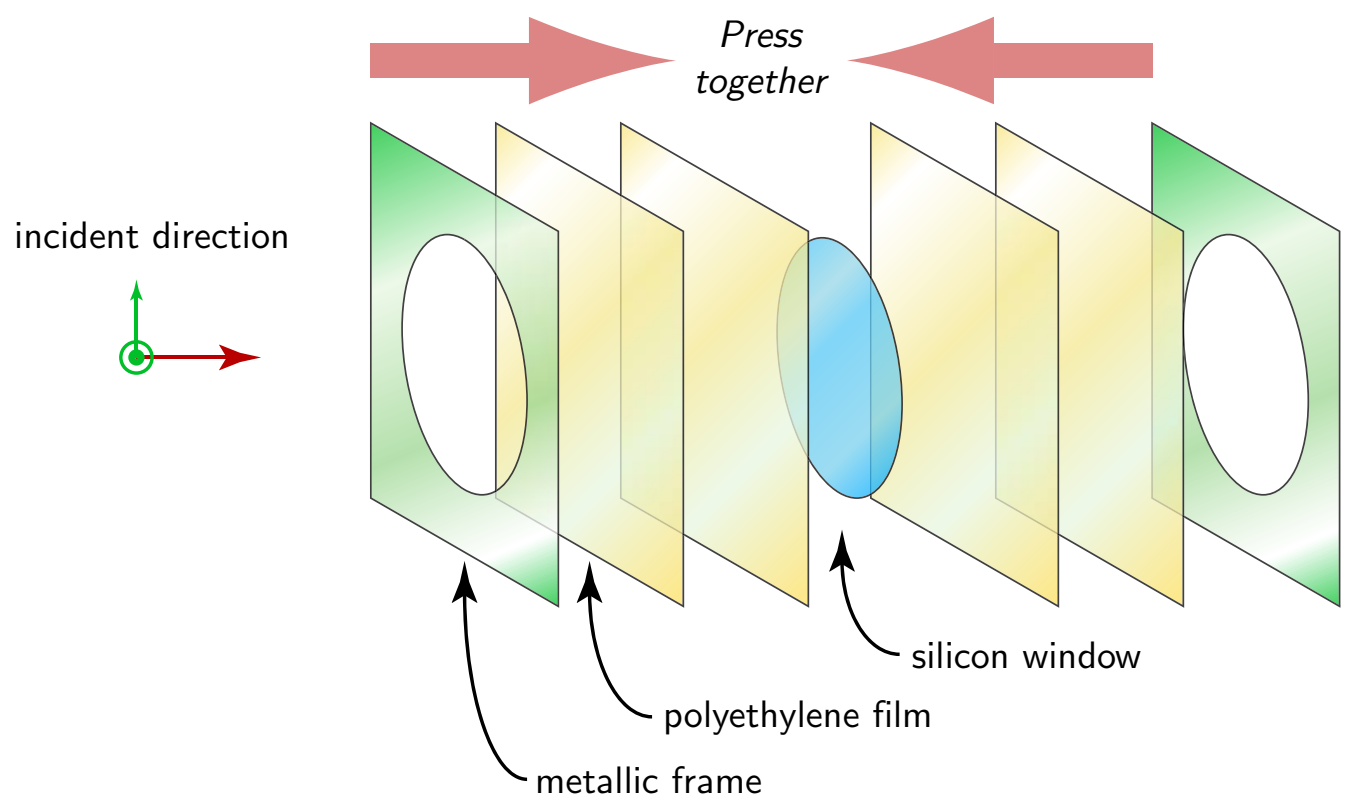

Figure 7. Retrofittable coating system. Multiple layers of polyethylene films are put together and pressed onto the silicon window by metallic frames. Combined together, these films perform as an antireflection coating for the silicon window.

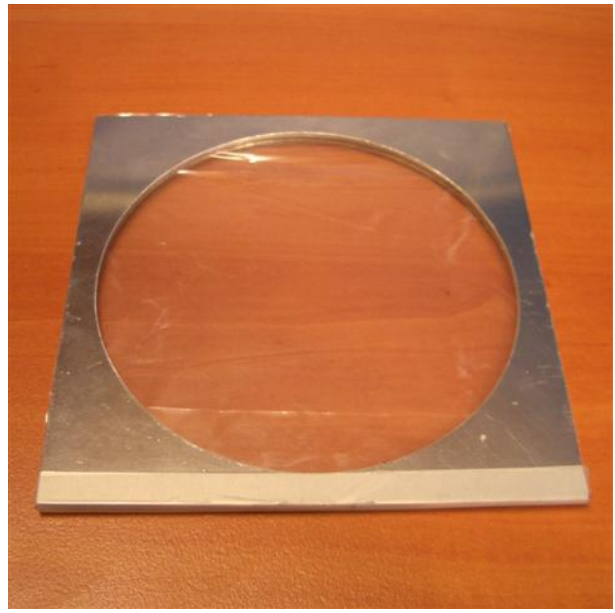

(a) PE frame without silicon window

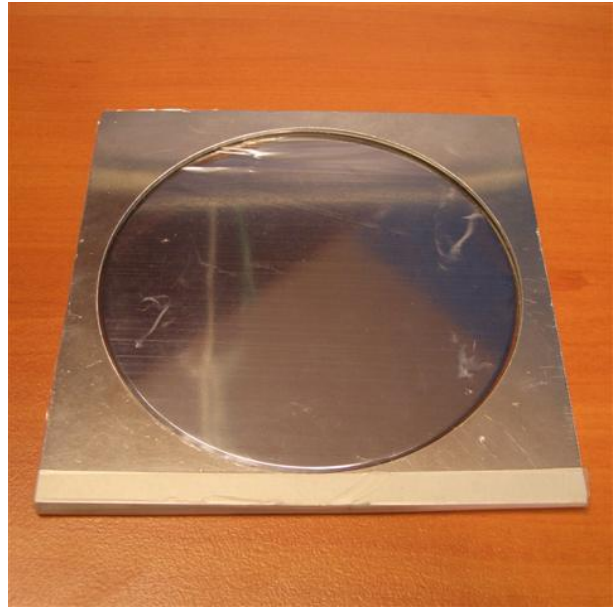

(b) PE frame with silicon window

Figure 8. Polyethylene frame. The structure of this frame is outlined in Figure 7.

\section{RETROFITTABLE ANTIREFLECTION SYSTEM}

Due to its common use in T-ray systems, a high-resistivity silicon wafer is used in this experiment. For the perfect transmittance, a coating material should have the index of refraction of $\sqrt{3.418}=1.85$. However, an ordinary material with the closest index is a series of polymers, which has the index of approximately 1.5 . This discrepancy is tolerable with a slightly lower antireflection performance.

Since the thickness of an antireflection coating for T-rays is in the order of tens of microns, it is possible to use a common polyethylene bag, which has a thickness ranging from ten to a hundred microns. Use of a pre-made polyethylene sheet as a window coating not only avoids a complex material deposition technique, but also opens up the possibility for retrofittable and interchangeable antireflection layers in T-ray systems. During a 
T-ray experiment, the window coating thickness can be rapidly changed to enhance the transmittance of desired frequencies.

In order to allow rapid change of a coating layer, a polyethylene film is stuck to a window by a supporting frame without adhesion, as demonstrated in Figure 7 and 8. Changing the coating thickness is simply carried out by replacing or adding polyethylene films.

In this paper, the antireflection performance is verified for two different coating thicknesses, $48 \pm 2.3$ microns and $103 \pm 10$ microns. The thicker layer is composed of two polyethylene sheets, one has the thickness of $48 \pm 2.3$ microns and the other has the thickness of $55 \pm 7.6$ microns. The coating is applied to both sides of the silicon window. According to the quarter-wave formula in Equation 9, and assuming the index of refraction of polyethylene is 1.5, the thin coating would be optimised for a central frequency, where the reflectance is zero, at $1.04 \mathrm{THz}$, and the thick coating would be for a central frequency at $0.49 \mathrm{THz}$.

\section{OPTICAL PROPERTIES OF WINDOW AND COATING}

Prior to testing for the transmittance of the coated windows, the optical properties of bulk materials, that will be used for the window and coating, are measured, as they possess some distinctive features induced by their production processes. A silicon wafer that will be used as a window, even thought it is high-resistivity, might have high absorption due to impurities. Polyethylene sheets somewhat differ in their optical characteristics, since the production process varies for each manufacturer.

\subsection{Silicon window}

Supplied by Siltronix, the window used in this experiment is an undoped $\langle 100\rangle$ CZ-grown silicon wafer polished on both sides with the thickness of $1.5213 \pm 0.0021 \mathrm{~mm}$ and the diameter of $10.2 \mathrm{~cm}$. Through THz-TDS, the optical constants in the frequency range from 0.2 to $1.0 \mathrm{THz}$ can be found, as shown in Figure 9 .

The measured index of refraction of our silicon is almost constant and comparable to the value of 3.418 of a FZ-grown silicon reported by Grischkowshy et al. ${ }^{17}$ However, the measured absorption coefficient is two orders of magnitude larger than that reported by Grischkowshy et al., ${ }^{17}$ in particularly at low frequencies. Since the wafer is polished on both sides, the scattering at the surfaces is negligible. Therefore, the high absorption could be attributed to impurities induced by the $\mathrm{CZ}$ method.
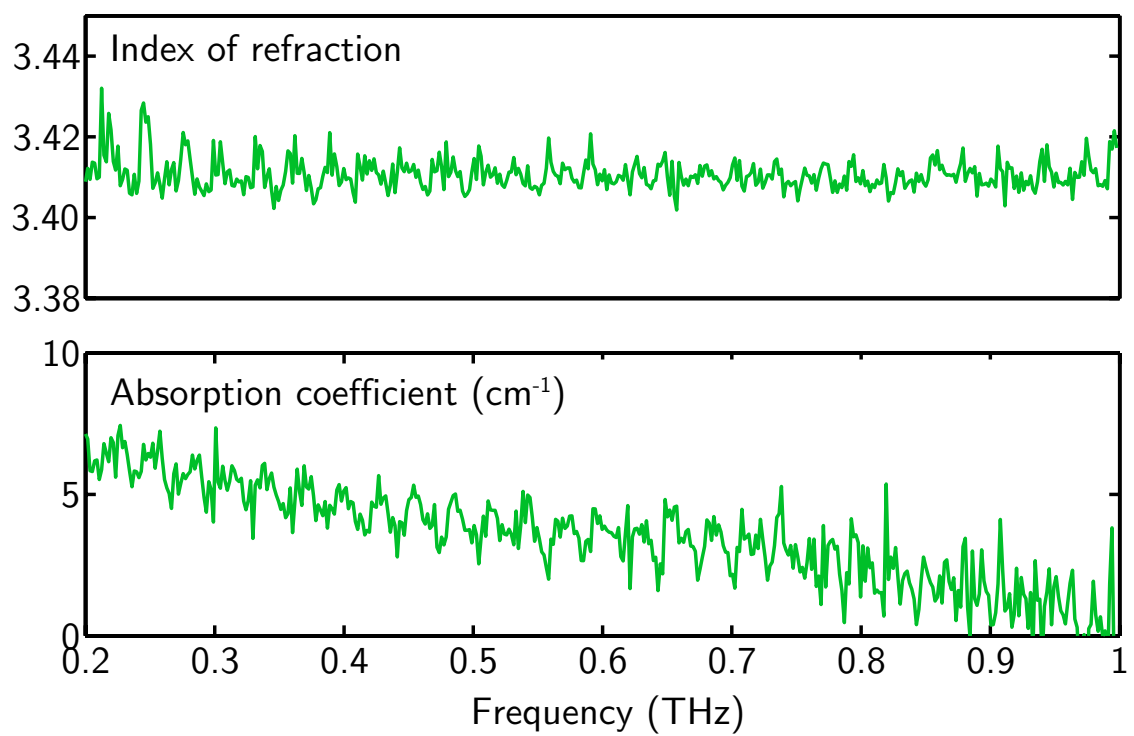

Figure 9. Optical constants of silicon. The index of refraction is constant over frequencies of interest, whereas the absorption coefficient is high at low frequencies. 


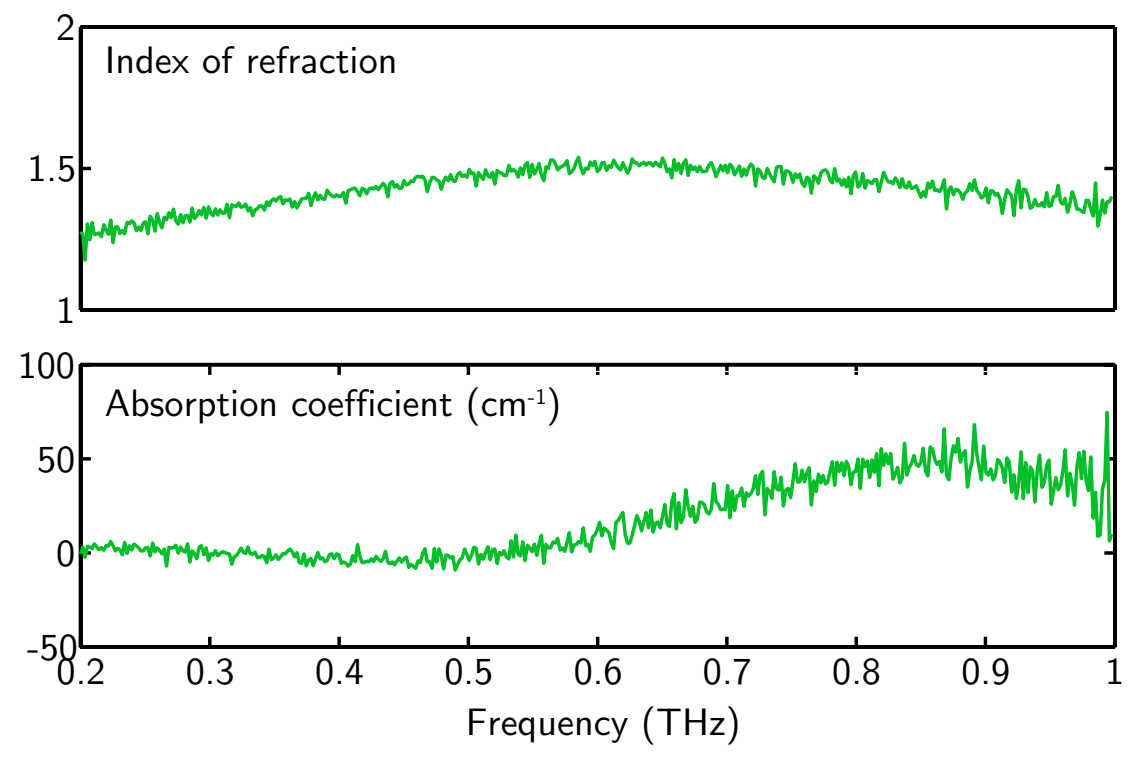

Figure 10. Optical constants of polyethylene. The index of refraction varies strongly with the frequency. The absorption coefficient is small from 0.2 to $0.5 \mathrm{THz}$, and increases rapidly after $0.5 \mathrm{THz}$.

\subsection{Polyethylene coating}

Two low-density polyethylene films, taken from common PE bags, with the total thickness of $0.0959 \pm 0.0046 \mathrm{~mm}$ are put together and measured for the optical constants in the T-ray frequency range. Note that the thickness variation of a low-graded polyethylene sheet is relatively large. This can affect a measurement of the optical constants and, furthermore, the efficiency of the coating.

Figure 10 shows the index of refraction and the absorption coefficient of polyethylene. In contrast with the value found by Birch et al. ${ }^{13}$ the measured index of refraction varies over the frequency range, rather than being fixed at 1.51. In addition, the absorption coefficient grows rapidly in the frequency range from 0.5 to $1.0 \mathrm{THz}$. Impurities, bubbles, and surface scattering could be associated with such high absorption.

\section{TRANSMITTANCE OF COATED WINDOWS}

Recall that two different polyethylene thicknesses, of 48 and 103 microns, are used as coatings. The transmittances of thinly-coated and thickly-coated windows are shown in Figure 11 and 12, respectively, in comparison with the transmittance of an uncoated window.

In the case of either thin or thick coating, the fluctuations after the main T-ray pulse prohibit removal of reflections in the time domain, and thus the oscillations exist in the transmittance spectra. In addition, the overall low transmittances of both coated and uncoated wafers are ascribed to the absorption of bulk silicon, grown by the $\mathrm{CZ}$ method. These do not impede interpretation, as long as we are interested in the relative transmittances of coated and uncoated wafers.

In the range of 0.7 to $1.0 \mathrm{THz}$, the thinly- or thickly-coated windows shows a lower transmittance than that of the uncoated window, because of the intrinsic absorption of the polyethylene sheets, which becomes large in this frequency range (see Figure 10). The high absorption of polyethylene prevents observation of the antireflection performance between 0.7 and $1.0 \mathrm{THz}$.

For the thinly-coated window, the transmittance in Figure 11 has an enhancement, broadly from 0.3 to 0.6 $\mathrm{THz}$, peaking at $0.45 \mathrm{THz}$. The peak transmittance position is in contradiction with the theoretical centre frequency at $1.04 \mathrm{THz}$. For the thickly-coated window, the spectrum in Figure 12 shows two transmittance enhancements at 0.3 and $0.6 \mathrm{THz}$. Again, these positions conflict with the expected centre frequency at $0.49 \mathrm{THz}$ and its odd harmonic at $1.47 \mathrm{THz}$. Even though the coating thickness variations are taken into consideration, 


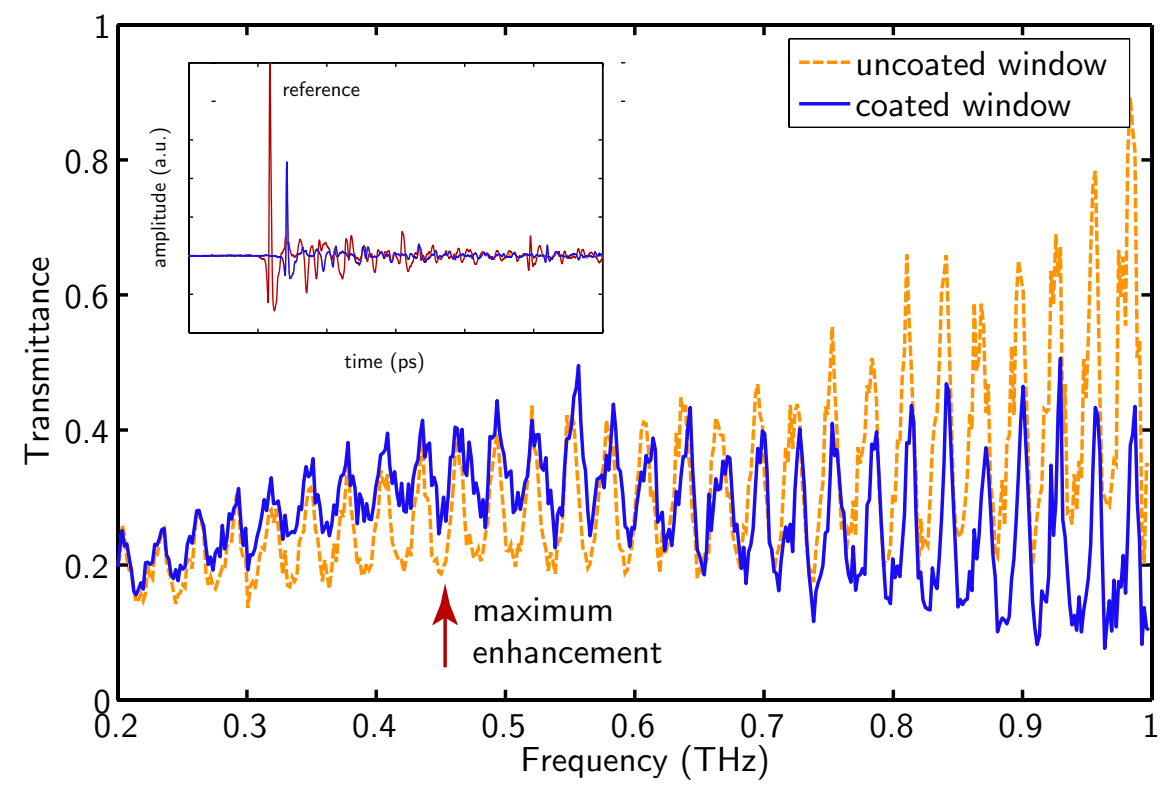

Figure 11. Measured transmittance of thin coating. The coated silicon window shows maximum transmittance enhancement at $0.45 \mathrm{THz}$. The inset shows the reference pulse and the pulses passing uncoated and coated windows.

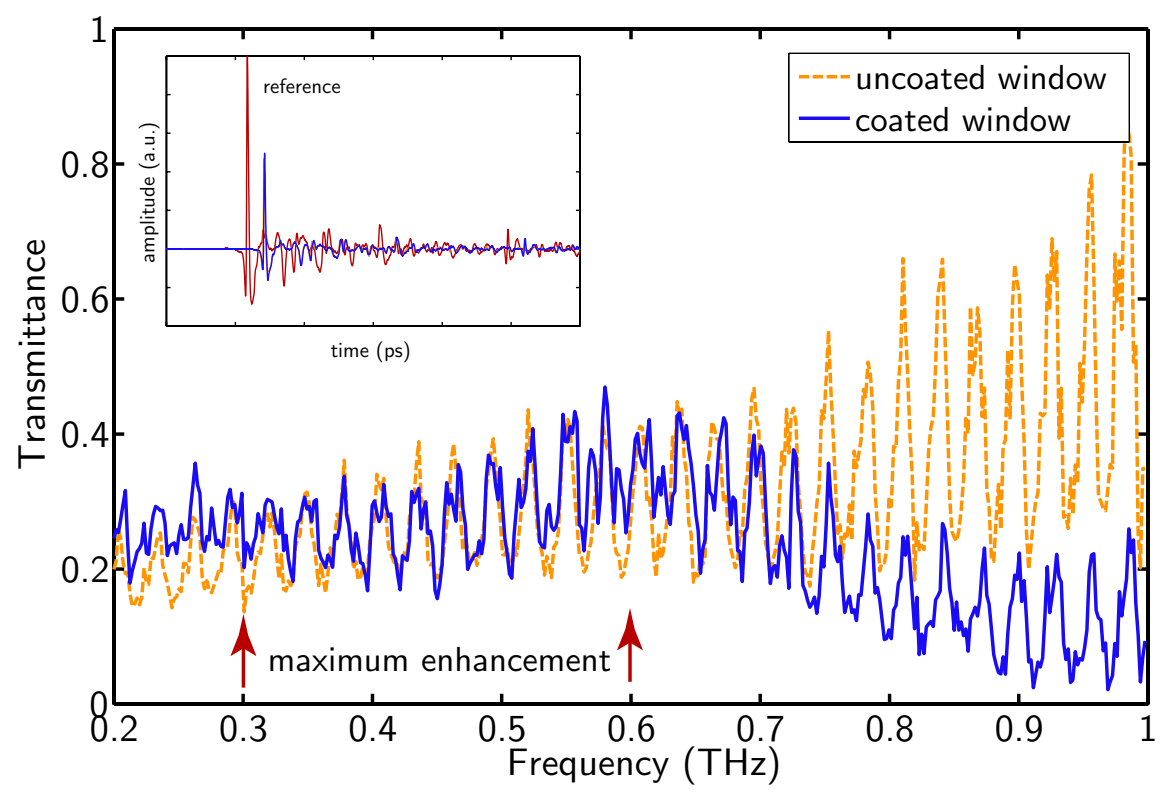

Figure 12. Measured transmittance of thick coating. The coated silicon window shows maximum transmittance enhancement at 0.3 and $0.6 \mathrm{THz}$. The inset shows the reference pulse and the pulses passing uncoated and coated windows.

the uncertainties in central frequency are not large enough to justify the measured results. The reason behind these inconsistencies will be discussed in the next section. 


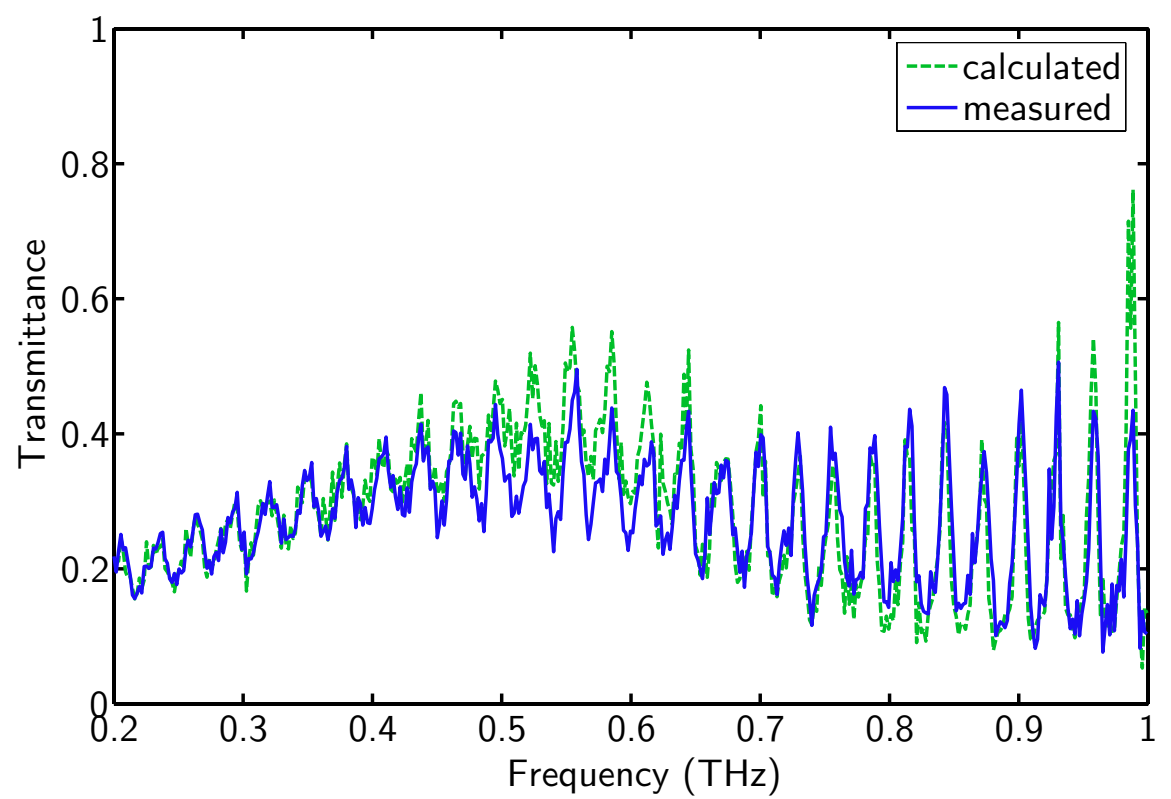

Figure 13. Calculated and measured transmittances of a thinly-coated silicon window. Two air gaps, each of which has the thickness of 45 microns, are inserted between the coating and the window for the calculated transmittance.

\section{COMPARISON OF MEASURED RESULTS TO MODELS}

From the previous section, it is obvious that the measured transmittances are far different from expectation. A possible reason is that air gaps might be present between the coatings and the window, since no adhesive nor vacuum is applied between them. (Note that our polyethylene sheets cannot support a vacuum.) In order to confirm this assumption, the characteristic matrix method is employed to simulate situations where narrow air gaps exist between the thin or thick coating and the window.

Figure 13 compares the calculated and measured transmittances of the thinly-coated window. In the simulation, two air gaps are set between the front and back coatings and the window, and the optical constants of silicon and polyethylene are taken from the measurement. The thickness of air gaps are varied in steps until the calculated transmittance is close to the measured transmittance. The theoretical thickness of each air gap is found to be around 45 microns.

Similarly, for the thick coating case, four air gaps are assumed between four polyethylene sheets and the window. The simulation gives the closest transmittance when the gap thickness is 65 microns. The comparison between the calculated and measured transmittances for the thick coating is shown in Figure 14

The calculation of the transmittances from the characteristic matrix method confirms that the implemented retrofitting antireflection has air gaps between the window and the coating. Though the antireflection is still effective, its operational frequencies are offset from the theoretically designed frequencies. With more accurate control of air gaps, this scheme might lead to the possibility of multilayer antireflection coatings.

\section{CONCLUSIONS}

A retrofittable antireflection coating for a transmission window operating with T-rays has been demonstrated. The retrofittable system allows rapid interchange of the coating thickness and coating material to accommodate desired frequencies. In the experiment, a silicon wafer was selected as window coated by various thicknesses of polyethylene sheets. Even though the index of refraction of polyethylene coating was not perfectly suited to the silicon window, the coating enhances the window transmittance to a promising degree. However, the operational frequencies of the coating are different from theoretical expectation. Through a characteristic matrix analysis, it was found that air gaps present between the coatings and the window can explain the discrepancy. These air 


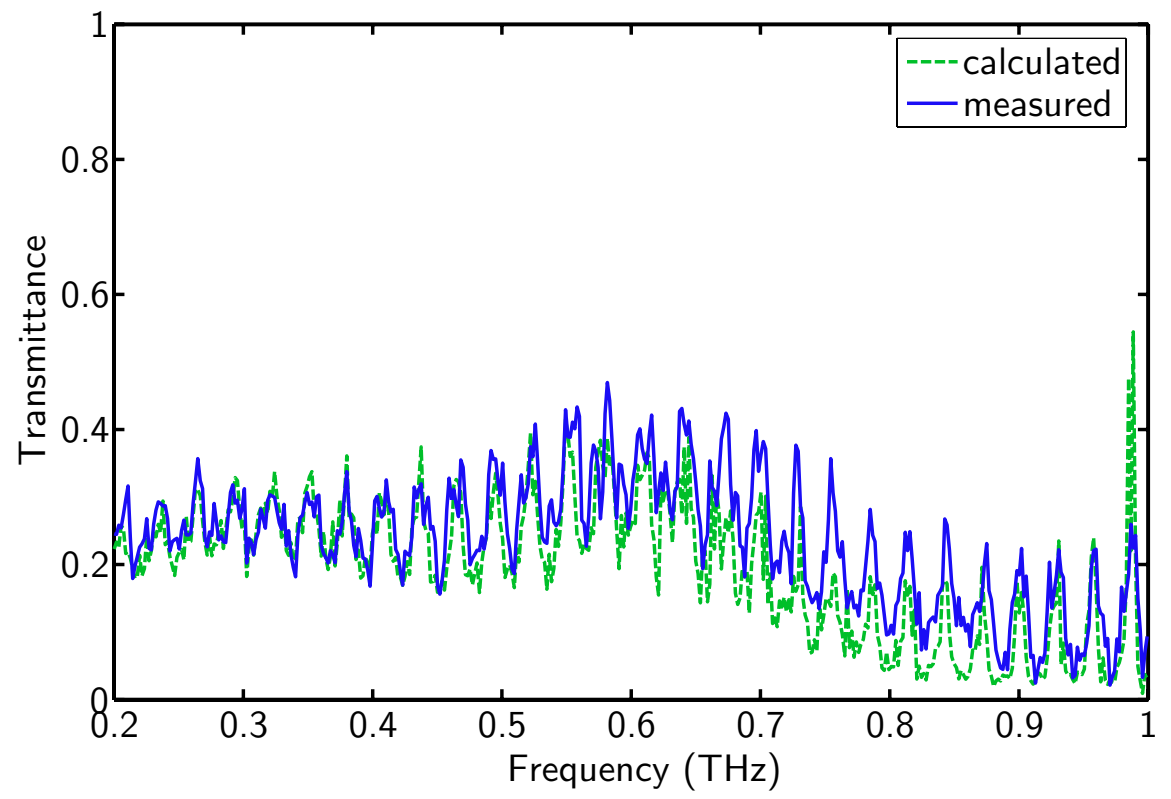

Figure 14. Calculated and measured transmittances of a thickly-coated silicon window. Four air gaps, each of which have a thickness of 65 microns, are inserted between the coating and the window for the calculated transmittance.

gaps, although difficult to remove, could probably be controlled in such a way to promote multilayer antireflection coatings.

\section{ACKNOWLEDGEMENTS}

The authors would like to thank Ian Linke for constructing the polyethylene frame. W. Withayachumnankul would like to thank Australian IPRS and University of Adelaide scholarships. The Adelaide T-ray program is supported by Australian Research Council and Sir Ross \& Sir Keith Smith Fund.

\section{REFERENCES}

1. S. P. Mickan, A. Menikh, H. Liu, C. A. Mannella, R. MacColl, D. Abbott, J. Munch, and X.-C. Zhang, "Label-free bioaffinity detection using terahertz technology," Physics in Medicine and Biology 47, pp. 37893795, 2002.

2. K. R. Armstrong and F. J. Low, "Far-infrared filters utilizing small particle scattering and antireflection coatings," Applied Optics 13(2), pp. 425-430, 1974.

3. K. Kawase and N. Hiromoto, "Terahertz-wave antireflection coating on Ge and GaAs with fused quartz," Applied Optics 37(10), pp. 1862-1866, 1998.

4. K. Kawase, N. Hiromoto, and M. Fujiwara, "Terahertz-wave antireflection coating on Ge wafer using optical lapping method," Electronics and Communications in Japan, Part 2 83(3), pp. 10-15, 2000.

5. C. R. Englert, M. Birk, and H. Maurer, "Antireflection coated, wedged, single-crystal silicon aircraft window for the far-infrared," IEEE Transactions on Geoscience and Remote Sensing 37(4), pp. 1997-2003, 1999.

6. A. J. Gatesman, J. Waldman, M. Ji, C. Musante, and S. Yngvesson, "An anti-reflection coating for silicon optics at terahertz frequencies," IEEE Microwave and Guided Wave Letters 10(7), pp. 264-266, 2000.

7. I. Hosako, "Antireflection coating formed by plasma-enhanced chemical-vapor deposition for terahertzfrequency germanium optics," Applied Optics 42(19), pp. 4045-4048, 2003.

8. I. Hosako, "Optical thin film technology used in the terahertz frequency," Journal of the National Institute of Information and Communications Technology 51(1), pp. 87-94, 2004. 
9. I. Hosako, "Multilayer optical thin films for use at terahertz frequencies: method of fabrication," Applied Optics 44(18), pp. 3769-3773, 2005.

10. T. Löffler, K. J. Siebert, N. Hasegawa, T. Hahn, G. Loata, R. Wipf, M. Kreß, M. Thomson, and H. G. Roskos, "Terahertz surface and interface characterization," in IEEE MTT-S International Microwave Symposium, pp. 633-636, 2005.

11. S. Biber, D. Schneiderbanger, L.-P. Schmidt, M. Walther, B. M. Fischer, M. Schwarzer, and P. U. Jepsen, "Low loss silicon window material for submillimeter waves using micromachined artificial dielectrics for anti-reflection coating," in Conference Digest of the Joint 29th International Conference on Infrared and Millimeter Waves and 12th International Conference on Terahertz Electronics, pp. 105-106, 2004.

12. E. Hecht, Optics, Addison-Wesley, 2 ed., 1987.

13. J. R. Birch, J. D. Dromey, and J. Lesurf, "The optical constants of some common low-loss polymers between 4 and $40 \mathrm{~cm}^{-1}$," Infrared Physics 21(4), pp. 225-228, 1981.

14. J. W. Lamb, "Miscellaneous data on materials for millimetre and submillimetre optics," International Journal of Infrared and Millimeter Waves 17(12), pp. 1997-2034, 1996.

15. G. J. Simonis, "Index to the literature dealing with the near-millimeter wave properties of materials," International Journal of Infrared and Millimeter Waves 3(4), pp. 439-469, 1982.

16. T. Ohba and S. Ikawa, "Far-infrared absorption of silicon crystals," Journal of Applied Physics 64(8), pp. 4141-4143, 1988.

17. D. Grischkowsky, S. Keiding, M. van Exter, and C. Fattinger, "Far-infrared time-domain spectroscopy with terahertz beams of dielectrics and semiconductors," Journal of the Optical Society of America B: Optical Physics 7(10), pp. 2006-2015, 1990.

18. E. V. Loewenstein, D. R. Smith, and R. L. Morgan, "Optical constants of far infrared materials. 2: Crystalline solids," Applied Optics 12(2), pp. 398-406, 1973.

19. B. M. Fischer, Broadband THz Time-Domain Spectroscopy of Biomolecules - A Comprehensive Study of the Dielectric Properties of Biomaterials in the Far-Infrared. PhD thesis, Department of Molecular and Optical Physics, Freiburg Materials Research Center, Universität Freiburg, 2005.

20. D. R. Smith and E. V. Loewenstein, "Optical constants of far infrared materials. 3: plastics," Applied Optics 14(6), pp. 1335-1341, 1975.

21. J. Shao and J. A. Dobrowolski, "Multilayer interference filters for the far-infrared and submillimeter regions," Applied Optics 32(13), pp. 2361-2370, 1993.

22. N. Krumbholz, K. Gerlach, F. Rutz, M. Koch, R. Piesiewicz, T. Kürner, and D. Mittleman, "Omnidirectional terahertz mirrors: A key element for future terahertz communication systems," Applied Physics Letters 88, p. 202905, 2006.

23. D. Turchinovich, A. Kammoun, P. Knobloch, T. Dobbertin, and M. Koch, "Flexible all-plastic mirrors for the THz range," Applied Physics A: Materials Science \& Processing 74, pp. 291-293, 2002.

24. D. A. DeCrosta, J. J. Hackenberg, and J. H. Linn, "Characterization of high oxygen:tetraethylorthosilicate ratio plasma-enhanced chemical vapor deposited films," Journal of the Electrochemical Society 143(3), pp. 1079-1084, 1996. 